Document downloaded from:

http://hdl.handle.net/10251/82439

This paper must be cited as:

Sendra, S.; García-García, L.; Jimenez, JM.; Lloret, J. (2017). Low-cost vehicle driver assistance system for fatigue and distraction detection. En Lecture Notes of the Institute for Computer Sciences, Social Informatics and Telecommunications Engineering. Springer Verlag. 69-78. doi:10.1007/978-3-319-51207-5_7 '

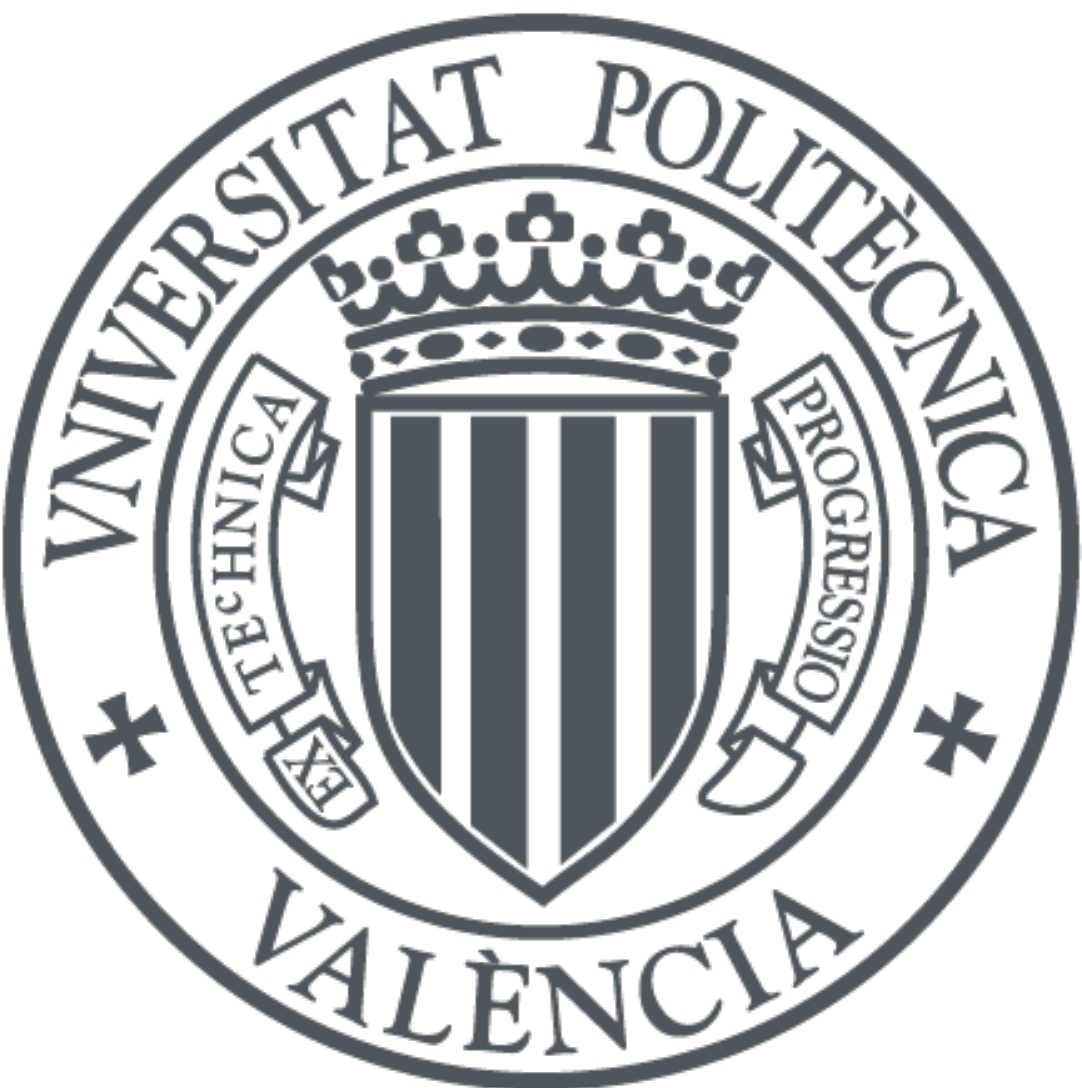

The final publication is available at

https://link.springer.com/chapter/10.1007/978-3-319-51207-5_7

Copyright Springer Verlag

Additional Information 


\title{
Low-Cost Vehicle Driver Assistance System for Fatigue and Distraction Detection
}

\author{
Sandra Sendra $^{1,2}$, Laura Garcia ${ }^{2}$, Jose M. Jimenez ${ }^{2}$, and Jaime Lloret $^{2}$ \\ ${ }^{1}$ Signal Theory, Telematics and Communications Department (TSTC), Universidad de \\ Granada, \\ C/ Periodista Daniel Saucedo Aranda, s/n. E-18071 Granada, Spain \\ ${ }^{2}$ Integrated Management Coastal Research Institute, Universidad Politecnica de Valencia, \\ C/Paraninf,1. 46730. Grao de Gandia, Spain \\ \{ssendra@ugr.es,laugarg2@teleco.upv.es,jojiher@dcom.upv.es,jlloret@dcom.upv.es\}
}

\begin{abstract}
In recent years, the automotive industry is equipping vehicles with sophisticated, and often, expensive systems for driving assistance. However, this vehicular technology is more focused on facilitating the driving and not in monitoring the driver. This paper presents a low-cost vehicle driver assistance system for monitoring the drivers activity that intends to prevent an accident. The system consists of 4 sensors that monitor physical parameters and driver position. From these values, the system generates a series of acoustic signals to alert the vehicle driver and avoiding an accident. Finally the system is tested to verify its proper operation.
\end{abstract}

Keywords: Low-cost sensors, Vehicular technology, Driver assistance system, Fatigue episodes, distraction detection, sensing system.

\section{Introduction}

As years go by, vehicular technology has been improving to satisfy the needs of its users. Most of this technology is based on acquiring data to improve the performance of the car and enhance its safety. To do this, cameras, sensors and Global Positioning System (GPS) technology are used. These devices are employed on active and passive safety systems [1] such as Antilock Brake System (ABS), Electronic Stability Control (ESP), power steering, airbags or seatbelts.

As it is shown in the annual report on road casualties provided by the Spanish Government [2], the number of victims in traffic accidents has decreased since 1989. The decrease of victims has happened even when the number of cars in the country has increased. One of the main reasons of the decrease in mortality is the usage of different kind of sensors that increase the safety of the car. Many of these sensors are created to alert the vehicle driver of an imminent collision [3].

Despite the decrease of traffic fatalities, the number of deaths in traffic accidents is quite disturbing. In 2014, the number of deaths caused by traffic accidents in Spain was 1.688 people [4]. Many deaths are caused by the intake of toxic substances such as alcohol or drugs. Even prescription drugs may generate side effects that affect 
driving. Fatigue can be caused by the aforementioned substances and sleep deprivation, hot weather or driving long distances, among others.

Fatigue presents a wide range of symptoms [5]. The driver's vision turns blurry and the blinking rate gets higher. It also affects the behavior causing anxiety and making the driver more irritable. Fatigue increases the number of movements the driver does to accommodate as well as other type of movements such as tapping the wheel. Finally, Fatigue increases the time the driver takes to react in a dangerous situation. For this reason, it is important to have systems able to detect the aforementioned symptoms. This can help to increase the driver's safety, but most of the currently developed solutions are focused on eye movement and face detection [6].

This paper presents a low-cost vehicle driver assistance system to detect episodes of fatigue and distraction in drivers.

The rest of the paper is organized as follows. Section 2 presents the related work. Section 3 explains the system operation and presents the algorithm used to detect and classify the alarm level. This section also shows the system tests. Finally, Section 4 presents the conclusion and future work.

\section{Related Work}

We can find lots of previous works about how to monitor and study fatigue in drivers and sleepiness while driving. This section shows some of these works.

In general, these works can be divided into two groups. On the one hand, we find proposals based on facial recognition. Sigari et al. [7] present an interesting review of driver face monitoring systems for fatigue and distraction detection where the general structure of these systems is discussed.

Kutila et al. [8] describes a facility for monitoring the distraction of a driver and presents some early evaluation results. They present a module that is able to detect the driver's visual and cognitive workload by fusing stereo vision and lane tracking data, running both rule-based and support-vector machine (SVM) classification methods. The module has been tested with data from a truck and a passenger car. The results show over $80 \%$ success in detecting visual distraction and a 68-86 \% success in detecting cognitive distraction, which are satisfactory results.

Rezaei et al. [9] present a research that develops optimum values of Haar-training parameters to create a nested cascade of classifiers for real-time eye status detection. They present the unique features of their robust training database that significantly influenced the detection performance. Their systems have been implemented and tested in real-world with satisfactory results.

Mbouna et al. [10] present visual analysis of eye state and head pose (HP) for continuous monitoring of alertness of a vehicle driver. The proposed scheme uses visual features such as eye index (EI), pupil activity (PA), and HP to extract critical information on no alertness of a vehicle driver. Using a support vector machine (SVM) classifies a sequence of video segments into alert or nonalert driving events. Their experimental results show that their proposed scheme offers high classification 
accuracy with acceptably low errors and false alarms for people of various ethnicity and gender in real road driving conditions.

Wahlstrom et al. [11] present a project that involves the use of a dashboard mounted camera to monitor the direction a driver is looking. They accomplish the project by using the Framework for Processing Video (FPV), developed at the University of Minnesota by Osama Masoud. Their software uses the relative positions of the eyes and pupils to make statements about the gaze direction.

Cherrat et al. [12] propose a multifunction system based on intelligent sensors and cameras. The system is mainly based on learning systems for face recognition based on advanced algorithms Viola and Jones, PCA and management of drivers profiles based on preferences to provide the following features: early detection of sleep, unconsciousness and poor driver behavior, security against theft of vehicles, driver comfort and control and sharing of traffic information in real time between the conductors.

On the other hand, there are some other works that use other techniques to detect fatigue episodes in drivers. In this sense, we can find works as the one, presented by Dong et al. [13] who review the state-of-the-art technologies for driver inattention monitoring such as distraction and fatigue. In their work, authors summarize these approaches by dividing them into the following five different types of measures: 1) subjective report measures; 2) driver biological measures; 3) driver physical measures; 4) driving performance measures; and 5) hybrid measures. Authors think that the hybrid measures are believed to give more reliable solutions compared with single driver physical measures or driving performance measures, because the hybrid measures minimize the number of false alarms and maintain a high recognition rate, which promote the acceptance of the system.

All of these solutions require powerful systems to process all the generated data. Our proposal tries to implement a very cheap solution that can be used in any kind of car. It is based on the measurement of physical parameters and its main task is generating a fast acoustic alarm to prevent the driver and avoid possible accidents.

\section{System Description}

When implementing a sensing system able to be used by everybody, we have to provide low-cost solutions. This section presents the design of our proposal. It is based on several sensors which are in charge of collecting data from the vehicle driver and generates alert signals to avoid the driver sleeps and may suffer an accident.

\subsection{Overall Explanation}

Our low-cost vehicle driver assistance system is based on economic devices and it has been designed to be used in any kind of car. A simple processing unit is in charge of collecting data from several sensors. From the gathered data, the system will generate a kind of alarm in order to prevent the driver.

As Fig. 1 shows, the car is endowed of various sensors. In this case, we use 2 pressure sensors and 2 temperature sensors installed in the steering wheel (duplicate 
system for monitoring both hands). It also includes a proximity sensor based on a LDR installed in the headrest which will detect if the driver separates the head a considerable distance to the headrest. If the received light is too big, this could imply that the driver is starting to get drowsy and can nodding off.

Our Low-cost vehicle driver assistance system contains a buzzer near to the ear driver that will generate an acoustic alarm in case of detecting a fatigue state and driver distraction.

Finally, we have installed 2 more elements. The first one is a shock sensor that detects collisions or sudden braking and a push-button placed on the steering wheel, to interact with the driver

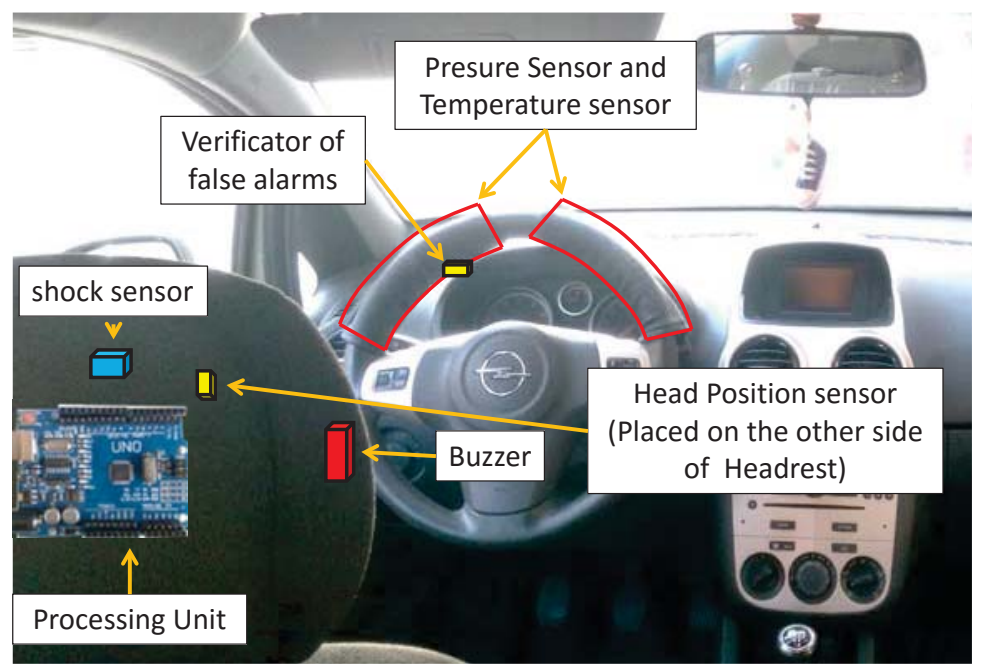

Fig. 1. Sensors placement inside a car.

The sensors are wired to the electronic board. The system performs the sensors' sampling every second and stores the results on a micro-SD card for possible postprocessing task, in case of accidents or future studies. The electronic board checks that the values collected by the sensors remain within the thresholds considered as normal. When any of these sensors exceeds any of these thresholds, the proposed algorithm (see Fig. 2) try to define whether it is a false alarm or sensors have registered a possible situation of the vehicle driver fatigue or distraction. 


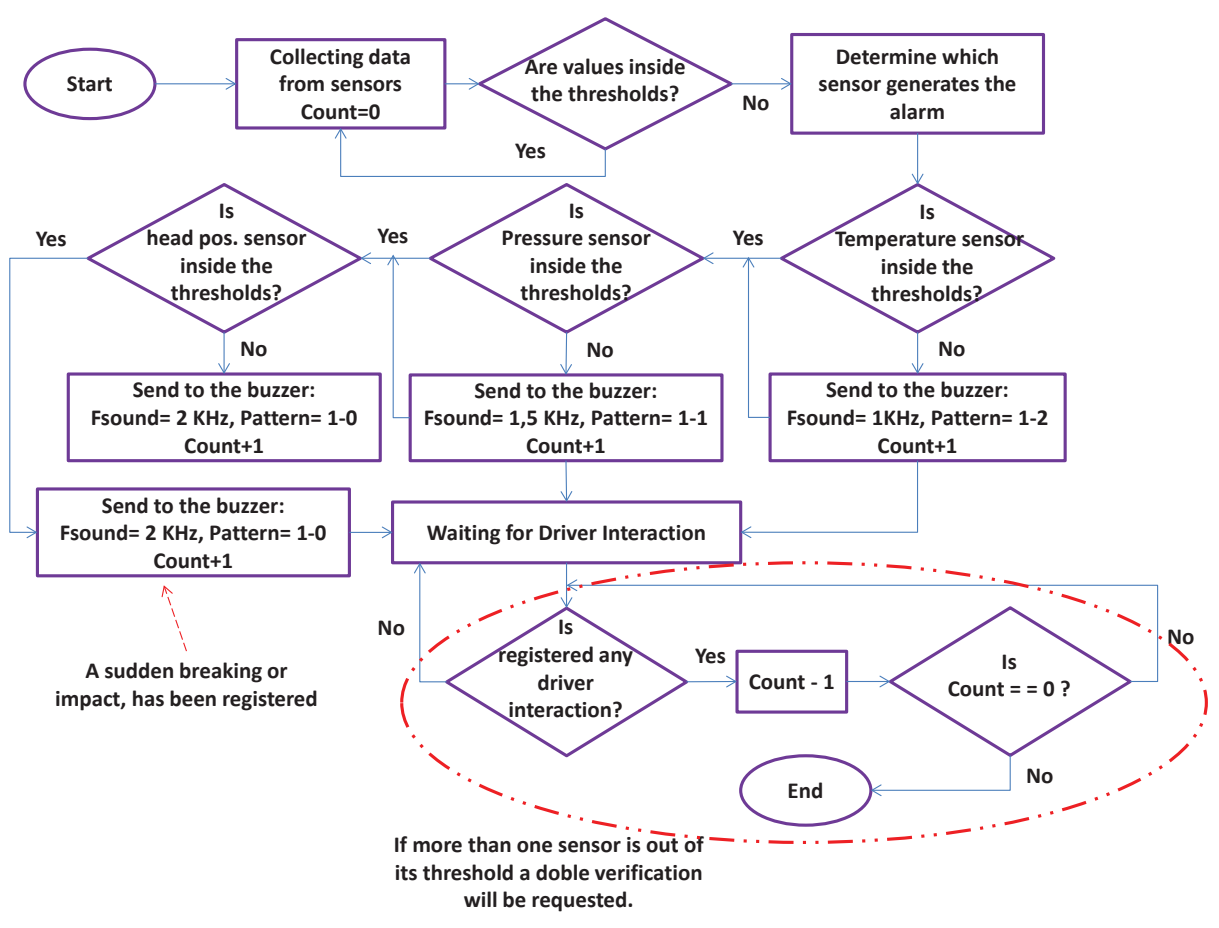

Fig. 2. Algorithm used by our low-cost vehicle driver assistance system for detecting fatigue and distraction episodes and generate the alarms.

As algorithm of Fig. 2 shows, the low-cost vehicle driver assistance system is continuously monitoring the sensors' activity. When it detects that some sensor has exceeded the specified thresholds, the system will try to define how many sensors are recording an anomalous behavior. As a function of this value, the system will generate a sound of a specific frequency and a pattern. For example, if the system detects that the temperature is the only parameter that has exceeded the threshold, the buzzer will emit a sound of $1 \mathrm{kHz}$ each 2 seconds. If the system detects that the temperature and pressure sensor have exceeded their thresholds, the buzzer will emit a sound of $1.5 \mathrm{kHz}$ each seconds. Finally, if all sensors have exceeded their thresholds, the buzzer will emit a continuous sound of $2 \mathrm{kHz}$. If a sudden breaking or impact has been registered the buzzer will also emit a continuous sound of $2 \mathrm{kHz}$.

To disable the alarm status and return the system to the sensing phase, the system requires the driver interaction by pushing the button placed on the steering wheel. If more than one sensor is out of its threshold a double verification will be requested.

\subsection{Processing Unit}

The system is based on a small electronic board based on the ATmega328 microprocessor. It has 14-pin digital inputs/outputs (6 of them can be used as PWM 
outputs), 6 analog inputs, a crystal oscillator of $16 \mathrm{MHz}$. This device can be powered from a computer via the USB connection or using batteries. Fig. 3 shows the processing unit. Unlike some previous models, this version allows to program the device without any FTDI USB-to-serial converter. In this case, the Board ONE uses a programmed Atmega16U2 chip which is used as a USB to serial converter.

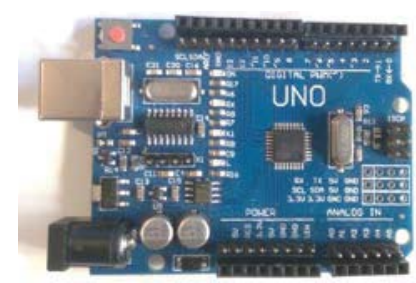

Fig. 3. Processing Unit used in the design of our Low-cost vehicle driver assistance system

\subsection{Sensors Used by the Low-Cost Vehicle Driver Assistance System}

The system is basically composed by 4 sensors and 2 actuators. This subsection presents the main features of each one.

Sensor for measuring the pressure of hands on the wheel. A Force Sensitive Resistor (FSR) [14] is a sensor that allows detecting and measuring physical pressure, squeezing and weight. This sensor is based on a resistor that changes its resistive value $(\Omega)$ depending on the pressure it registers.. However, FSRs are not indicated to accurate measurements because its accuracy can vary up to $10 \%$ from sensor to sensor. So, in our case, the FSR is used to detect a variation that exceeds a threshold. Fig. 4a shows the FSR sensor. The FSR 402 is able to support values of pressure from 0 to 100 Newtons which correspond to a resistance range from Infinite/open circuit (absence of pressure) to $100 \mathrm{~K} \Omega$ (light pressure). Since its behavior is like a resistor, it can be used as a part of a resistive voltage divider. In this way, the processing unit will register this pressure variation as a voltage value. Fig. 4b shows the output voltage of this sensor when it is combined with a resistance of $1 \mathrm{kOhm}$ and a power supply of $3.3 \mathrm{~V}$.

Temperature sensor: To develop the temperature sensor, it is used a resistance temperature detector (RTD). It is a resistance based on the variation of the resistance of a conductor with the temperature sensor. (See Fig. 5). Their behavior is very simple. When heating a metal, it is generated a higher thermal agitation that provokes the dispersion of more electrons reducing its average speed. This causes that resistance increase. The behavior of this sensor can be modeled by Eq. 1, where $\mathrm{R}$ is the value of resistance in $(\Omega), R_{0}$ is the resistance at reference temperature $T_{0}, \Delta t$ is the temperature deviation with respect to $T_{0}$ and $\alpha$ is the is the conductor temperature coefficient at $0{ }^{\circ} \mathrm{C}$. This sensor presents a linear behavior in wide temperature ranges. 


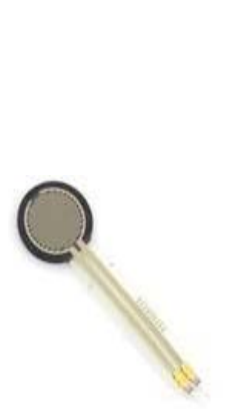

a

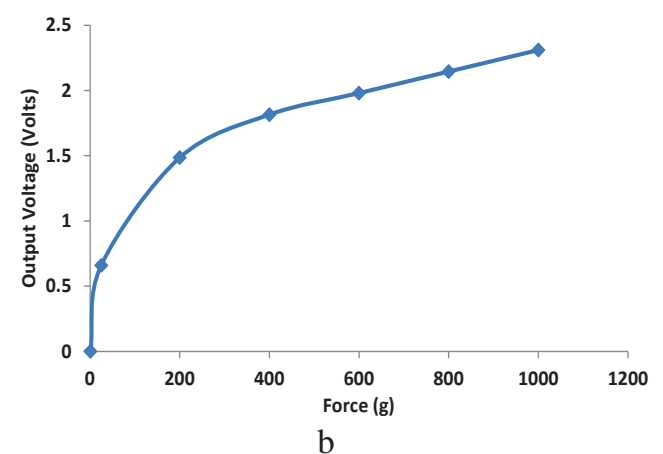

Fig. 5. PT100

Fig. 4. Force Sensitive Resistor and its behavior.

Head Position Sensor: This sensor is based on a light-dependent resistor (LDR). In this case, its resistance decreases when increasing incident light intensity. We can use this sensor combined with other resistance forming a voltage divider. If we use the LDR placed at the bottom of the voltage divider, it will give us the maximum voltage when the LDR is in total darkness, because it is having the maximum resistance to the current flow. In this situation registers the maximum value. However, if the LDR is placed at the top of the voltage divider, the result is the opposite.

Fig. 6 shows the head position sensor and its position on the headrest. The particularity of this small board is that it allows controlling the sensitivity of this sensor.
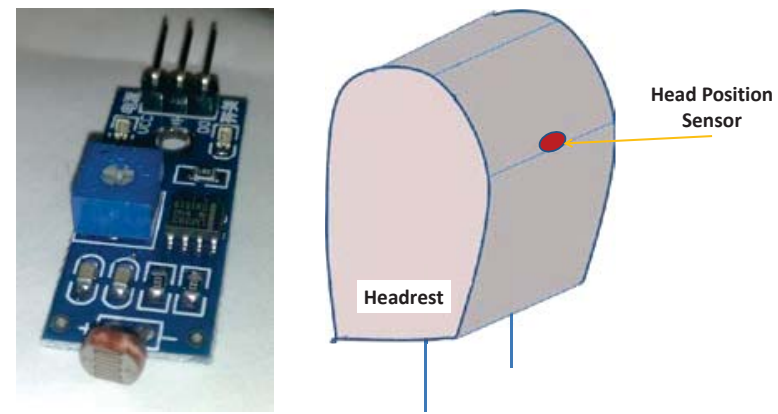

Fig. 6. Head Position sensor and its position in the Headrest

Shock Sensor: It is based on the Gaoxin SW-18010P vibration switch and allows using our system to detect impacts, shocks or shaking. When the sensor detects a jolt, the sensor generates a low level output signal. Fig. 7 shows the module used in this design. The sensor consists of a terminal that forms a center post and a second terminal that is a spring that surrounds the center post. If a sudden breaking or impact with sufficient force is transferred to the sensor, the terminal consisting of the spring moves and shorts both terminals together. The connection between the terminals is 
short but the central unit is able to detect this breakdown voltage. The sensor position is also important. In our case, it is place in the forward direction of the vehicle.

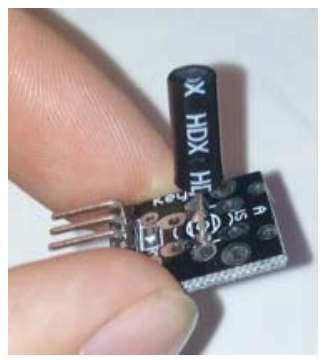

Fig. 7. Shock sensor

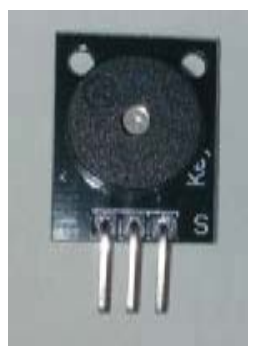

Fig. 8. Buzzer module placed near to the driver's ear.

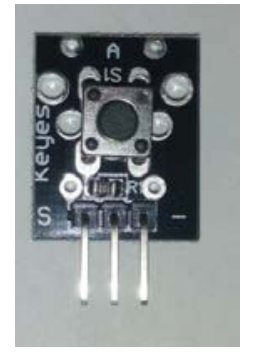

Fig. 9. Push-button for alarm verification

In order to alert the driver, our low-cost vehicle driver assistance system contains a buzzer. It is a KY-006 Small passive buzzer module (See Fig. 8). It is composed by a piezo-resistive element and a resonance box that permit generating audio signals from $1.5 \mathrm{kHz}$ to $2.5 \mathrm{kHz}$. As Fig. 1 showed, this element is placed near to the driver's ear. It is used as an alarm element which is in charge of generating an acoustic alarm to alert the driver if a fatigue status of distraction is detected. The output frequency can be configured by programing.

The last important element is a verification system. It is a simple push-button that requires the driver interaction. After detecting a dangerous situation, our driver assistant system will emit an acoustic alarm to alert the driver and prevent he/she sleeps while driving. In order to restore the system which implies that the driver is paying attention to the road, the driver has to push this button. After that, the system will go back to the sensing phase during which it is collecting data.

\subsection{System Simulation}

Finally, it is interesting to check if the system is able to detect the sensors value and generate the correct alarms. To check it, we have tested the system during 4 minutes. During this time, we have forced the sensors to change their values (See Fig. 10) and to exceed the correspondent threshold. As we can see in Fig.11, the low-cost vehicle driver assistance system is registering the alarm level and as the user pushes the button to disable the alarm, the alarm level decreases.

\section{Conclusion and Future Work}

When implementing a sensing systems and we want to offer these systems to as many people as possible, it is needed to think with low-cost system that allows developing accurate designs. This paper presents a low-cost vehicle driver assistance system for detecting fatigue episodes and distraction situations of a vehicle driver. 


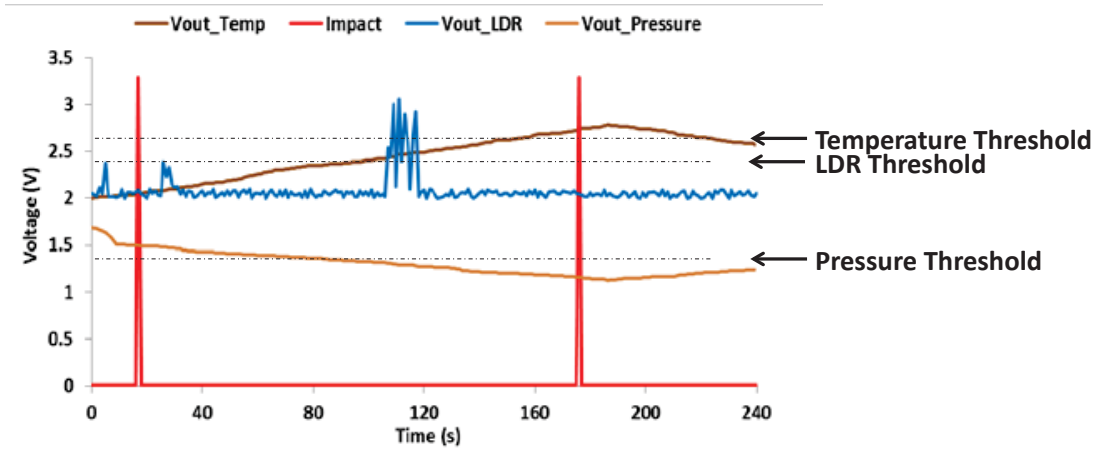

Fig. 10. Sensors monitoring

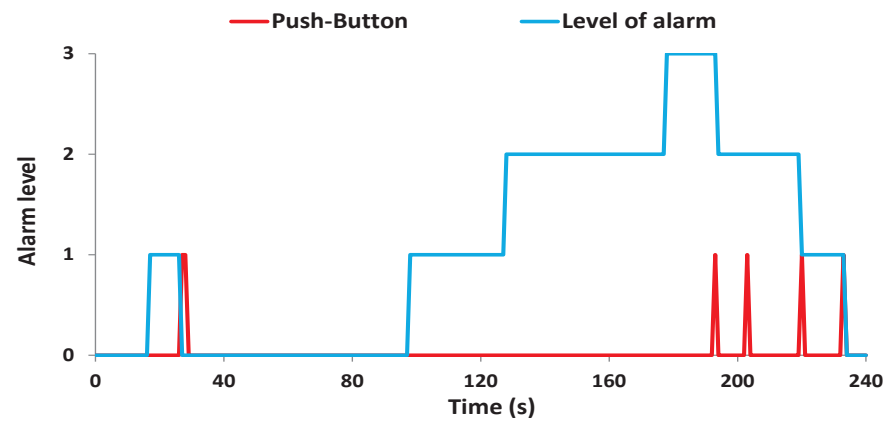

Fig. 11. Alarm generation as a function of sensors value

The system is based on an electronic board that acts as processing unit and 4 sensors (person temperature, pressure on the steering wheel, a light sensor placed on the headrest and a shock sensor that detect sudden breakings and impacts). The lowcost vehicle driver assistance system is able of classifying the alarms as a function of the number of sensors that have registered values out of the thresholds. It also contains 2 actuators, i.e., a buzzer and a push-button for interacting with the driver. After generating an alarm the driver has to disable it pushing the button.

This is the initial version of prototype. In future works, we want to include a small Global Positioning System (GPS) receiver to gather the vehicle position and a wireless technology module to interact with the smartphones in order to create a more autonomous system able to generate an emergency call via the smartphone. Finally, we would like to integrate the system in a more complex and energy efficient network [15][16] with the rest of sensors embedded in a car to generate more accurate responses.

Acknowledgments. This work has been partially supported by the "Programa para la Formación de Personal Investigador - (FPI-2015-S2-884)" by the“Universitat Politècnica de València”. 


\section{References}

1. Mapfre Foundation, (Online Article) Seguridad activa y pasiva. Available at: www.seguridadvialenlaempresa.com/seguridad-empresas/actualidad/noticias/seguridad-vialactiva-y-pasiva-2.jsp [Last access: Augus 25, 2016]

2. Dirección general de tráfico, Ministerio del Interior, Spanish Government, (Online Article) Las principales cifras de la siniestralidad vial. España 2014, pp. 21. Available at: http://www.dgt.es/es/seguridad-vial/estadisticas-e-indicadores/publicaciones/ [Last access: Augus 25, 2016]

3. Fukuhara H., Vehicle collision alert system, (Patent) US 5355118 A, October 11, 1994.

4. Dirección general de tráfico, Ministerio del Interior, Spanish Government, (Online Article) Anuario estadístico de accidentes 2014, pp. 10. Available at http://www.dgt.es/es/seguridadvial/estadisticas-e-indicadores/publicaciones/anuario-estadistico-general/ [Last access: Augus 25, 2016]

5. Dirección general de tráfico, Ministerio del Interior, Spanish Government. (Online Article) Otros factores de riesgo: La fatiga: .Available at: http://www.dgt.es/PEVI/documentos/catalogo_recursos/didacticos/did_adultas/fatiga.pdf [Last access: Augus 25, 2016]

6. Seeing machines web page. Available at: https://www.seeingmachines.com/ [Last access: Augus 25, 2016]

7. Sigari M. H., Pourshahabi M. R., Soryani M., Fathy M., A Review on Driver Face Monitoring Systems for Fatigue and Distraction Detection, International Journal of Advanced Science and Technology, Vol.64 (2014), pp.73-100, http://dx.doi.org/10.14257/ijast.2014.64.07

8. Kutila M., Jokela M, Markkula G., Romera Rue M., Driver Distraction Detection with a Camera Vision System, 14th IEEE International Conference on Image Processing (ICIP 2007), 16-19 Sept. 2007, San Antonio, TX, USA, DOI: 10.1109/ICIP.2007.4379556

9. Rezaei M., Klette R., 3D Cascade of Classifiers for Open and Closed Eye Detection in Driver Distraction Monitoring, Computer Analysis of Images and Patterns (CAIP 2011), Volume 6855 of the series Lecture Notes in Computer Science, pp 171-179 (2011)

10.Mbouna R. O., Kong S. G., Chun M. G, Visual Analysis of Eye State and Head Pose for Driver Alertness Monitoring, IEEE Transactions on Intelligent Transportation Systems, Volume: 14, Issue: 3, Sept. 2013, pp. 1462-1469, DOI: 10.1109/TITS.2013.2262098

11.Wahlstrom E., Masoud O., Papanikolopoulos N., Vision-based methods for driver monitoring, Proc. Int. Conf. Intelligent Transportation Systems, vol. 2, pp. 903-908, 2003

12.Cherrat L., Ezziyyani M., EL Mouden A, Hassar M., Security and Surveillance System for Drivers based on User Profile and Learning Systems for Face Recognition, Network Protocols and Algorithms, Vol 7, $\quad$ No 1, pp. 98 - 118,(2015), DOI: http://dx.doi.org/10.5296/npa.v7i1.7151

13.Dong Y., Hu Z., Uchimura K., Murayama N., Driver Inattention Monitoring System for Intelligent Vehicles: A Review, IEEE Transactions on Intelligent Transportation Systems, Volume: 12, Issue: 2, June 2011, pp. 596 - 614, DOI: 10.1109/TITS.2010.2092770

14.Force Sensitive $\underline{\text { Resistor features. Available }}$ at: http://www.trossenrobotics.com/productdocs/2010-10-26-DataSheet-FSR402-Layout2.pdf [Last access: August 25, 2016]

15.Louiza M., Samira M., A New Framework for Request-driven Data Harvesting in Vehicular Sensor Networks. Network Protocols and Algorithms, 2013, Vol 5, No 4. Pp.1-18.

16. Yao H., Si P., Yang R., Zhang Y. Dynamic Spectrum Management with Movement Prediction in Vehicular Ad Hoc Networks Ad Hoc \& Sensor Wireless Networks, 2016, Vol. 32, Num- 1-2:p. 79-97. 